\title{
Ultra-Wideband Low Noise Amplifiers For The Next Generation Very Large Array
}

\author{
Jose E. Velazco, Luis Ledezma, James Bowen, Lorene Samoska, \\ Melissa Soriano, Ahmed Akgiray, Sander Weinreb, and Joseph Lazio \\ Jet Propulsion Laboratory, California Institute of Technology \\ 4800 Oak Grove Dr. \\ Pasadena, CA 91109 \\ 818-354-4605 \\ jose.e.velazco@jpl.nasa.gov
}

\begin{abstract}
We have implemented a single wideband receiver package that could cover the 8 to $48 \mathrm{GHz}$ frequency range of the ngVLA. The current JVLA covers this frequency range employing five distinct receiver packages. We estimate that reducing the number of receiving systems required to cover the full frequency range should reduce operating costs. The receiver package we developed consists of a quad-ridge feed horn, low-noise amplifiers (LNA), and a down-converter to ana$\log$ intermediate frequencies. Both the feedhorn and the LNA are cryogenically cooled. On the LNA front we pursued two 8$48 \mathrm{GHz}$ MMIC designs, the first using 70-nm gallium arsenide, metamorphic high-electron-mobility-transistors (HEMT), and the second using 35-nm indium phosphide HEMTs. In this paper, we report the measured gain and noise temperatures of these LNAs.
\end{abstract}

\section{Table of Contents}

1. INTRODUCTION...............................

2. Low-Noise AMPLIFIER DESIGN ................2

3. NOISE BUDGET ..............................4

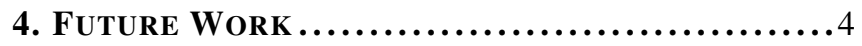

5. CONCLUSION .................................4

ACKNOWLEDGMENTS ............................4

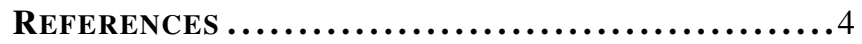

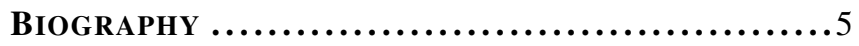

\section{INTRODUCTION}

The next-generation Very Large Array (ngVLA), is a concept for a radio astronomical interferometric array that will provide large improvements in sensitivity and angular resolution over existing telescopes such as the Jansky Very Large Array (JVLA) and the Atacama Large Millimeter/submillimeter Array (ALMA). The ngVLA will operate in the 1.2 to 116 $\mathrm{GHz}$ frequency range and its design is aimed at reducing operational and maintenance costs. The concept includes about $21418-\mathrm{m}$ reflector antennas and baselines up to 1000 $\mathrm{km}$ with a dense core on few-km scales for high surface brightness imaging, centered at the current JVLA site in New Mexico [1].

JPL is in a unique position both to contribute to the ngVLA and to benefit from it. With its frequency coverage, the ngVLA would cover all of the deep space communication frequency allocations and all of the planetary radar frequency allocations. Figure 1 shows a preliminary estimate of effective collecting area as a function of frequency for various

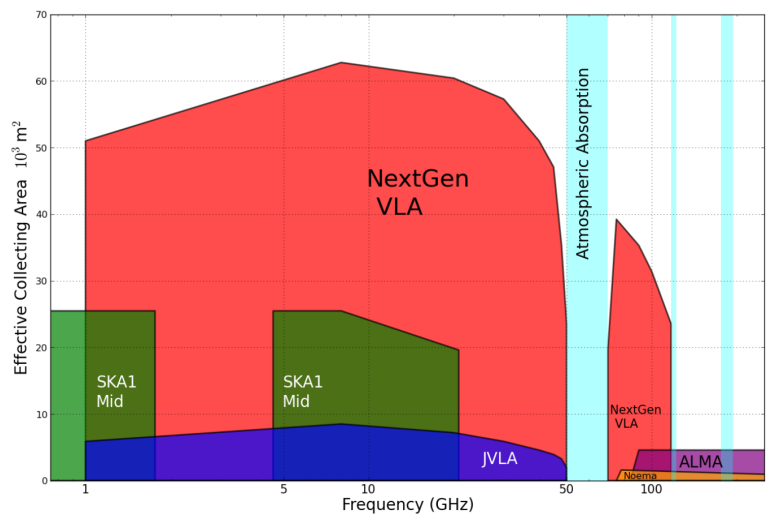

Figure 1. Preliminary estimate of effective collective area as a function of frequency for various telescopes (courtesy NRAO).

telescopes, including the proposed ngVLA.

The ngVLA could be used by JPL/NASA during key critical spacecraft events such as entry, descent, and landing or orbit insertions for missions at the outer planets or their icy moons. Historically, radio astronomy antennas have been used in the past to support such events. For example, the Very Large Array was used in conjunction with the DSN Goldstone 70$\mathrm{m}$ and 34-m antennas during the Voyager Neptune encounter [3]. The Green Bank observatory was used to receive direct to earth transmissions from The Mars Phoenix Lander during its entry, descent, and landing [4].

This effort focuses on the development of a prototype ultrawideband feed-receiver package for the ngVLA. Much like the case for the DSN, operational costs are increasingly recognized as a factor in determining the viability for current and future radio telescopes. For the ngVLA to achieve the required sensitivity, the feed-receiver systems will have to be cryogenically cooled, but in order to minimize operational costs associated with the cryogenics, the number of such feed-receiver systems should be minimized. Similarly, integrated and easily serviceable feed-receiver systems are expected to reduce maintenance costs and downtime. These considerations lead us to development of an integrated, cryogenically-cooled feed-receiver package with a continuous instantaneous frequency coverage of $8-48 \mathrm{GHz}$ and a system noise temperature that meets the requirements of the priority science programs of the ngVLA, $34 \mathrm{~K}$ at $10 \mathrm{GHz}$ and $45 \mathrm{~K}$ at $30 \mathrm{GHz}$ [2]. The receiver should be low cost, easy to manufacture and easy to service. In collaboration with other groups developing prototype systems for the ngVLA, we seek 
Table 1. $T_{\mathrm{RX}}$ vs Frequency for JVLA Receiver Bands

\begin{tabular}{|c|c|c|c|c|c|}
\hline Band & $\mathbf{X}$ & $\mathbf{K u}$ & $\mathbf{K}$ & $\mathbf{K a}$ & $\mathbf{Q}$ \\
\hline \hline Freq $(\mathrm{GHz})$ & $8-12$ & $12-18$ & $18-26.5$ & $26-40$ & $40-50$ \\
\hline$T_{R X}$ & 20 & 25 & 34 & 40 & 48 \\
\hline
\end{tabular}

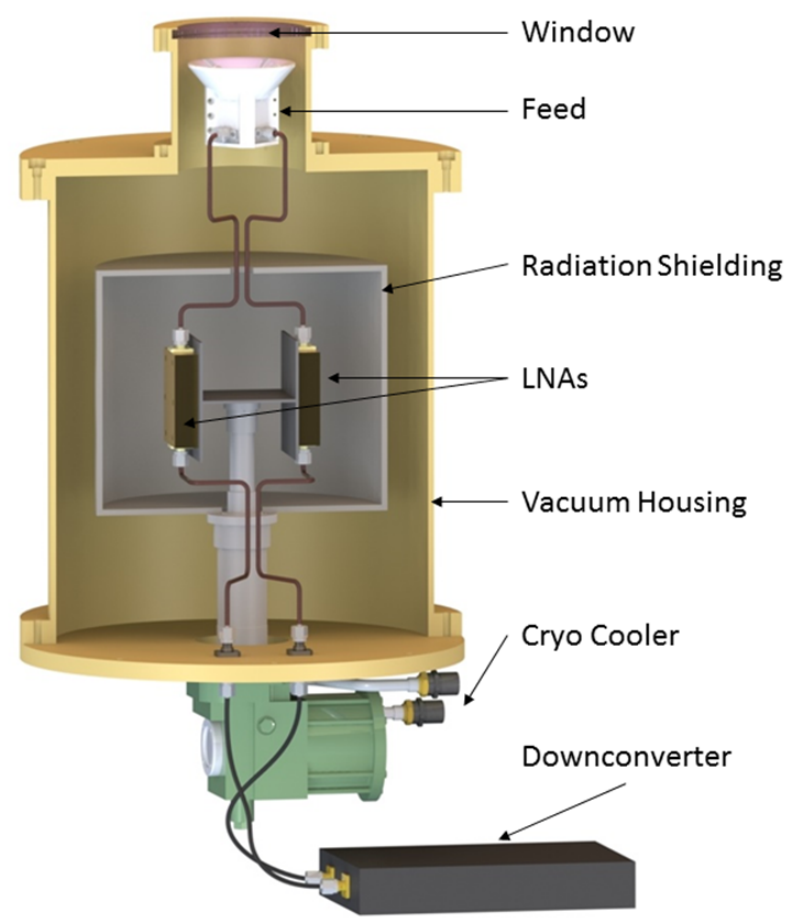

Figure 2. Ultra-wideband receiver concept.

to demonstrate that key components are sufficiently mature in time for the project to be included in the 2020 Astronomy Decadal Survey.

The DSN and current radio telescope receiving systems define the state-of-the-art in receivers. DSN receivers have extremely low system temperatures, typically $25 \mathrm{~K}$ or better, but in fairly narrow bands (mostly at S-, X-, and $\mathrm{Ka}$ bands) with typical bandwidths no larger than $10 \%$ [5]. The JVLA, operated by the NRAO, currently utilizes five different receiving systems for the $8-49 \mathrm{GHz}$ frequency range. The JVLA receiver frequency bands and their corresponding noise temperatures, $T_{R X}$, are shown in Table 1 .

The ultra-wide band receiver package, shown schematically in Figure 2, includes a feed, low-noise amplifiers and a down-conversion stage (the feed and LNA are cryo-cooled). Responding to the combination of scientific and operational considerations, our design goals for the receiver system were:

(1) Continuous frequency coverage of $8-48 \mathrm{GHz}$;

(2) Down-conversion stage with multiple bands providing an intermediate frequency whose value is dependent on the number of bands selected;

(3) System noise temperature of $34 \mathrm{~K}$ at $10 \mathrm{GHz}$ and $45 \mathrm{~K}$ at $30 \mathrm{GHz}$ as required by the ngVLA primary science programs [2];

(4) $30 \mathrm{~dB}$ gain across each of intermediate bands.

The goal was to have an integrated, cryogenically-cooled
Table 2. NGVLA Receiver LNA Requirements

\begin{tabular}{|c|c|c|}
\hline Parameters & Freq (GHz) & Requirement \\
\hline \hline$T_{e}$ (Noise & $8-40$ & $\leq 12 \mathrm{~K}$ \\
Temperature) & $40-48$ & $\leq 20 \mathrm{~K}$ \\
\hline Gain & $8-48$ & $\geq 30 \mathrm{~dB}$ \\
\hline Gain Flatness & $8-48$ & $\leq 6 \mathrm{~dB}$ \\
\hline Input Match & $8-15$ & $\leq-5 \mathrm{~dB}$ \\
Input Match & $15-48$ & $\leq-10 \mathrm{~dB}$ \\
\hline Output Match & $8-48$ & $\leq-10 \mathrm{~dB}$ \\
\hline
\end{tabular}

feed-receiver package with a sensitivity performance comparable to current narrower band systems on radio telescopes and the DSN but with a design that responds to the requirements of lower long-term operational costs.

In this paper we focus on the results of the wideband LNA's. In Section 2, we describe the design philosophy, fabrication, and test of the wideband monolithic microwave integrated circuit (MMIC), low-noise amplifiers ( L NAs). In S ection 3, we provide an analysis of noise temperature budget of the entire ultra-wide band receiver package consider the LNA results obtained. Future work is described in Section 4.

\section{LoW-Noise AMPLIFIER DESIGN}

We have developed custom Monolithic Microwave Integrated Circuits (MMICs) for the ultra-wideband ngVLA receiver package. A list of our LNA requirements is shown in Table 2. These represent aggressive goals based on the state of the art as well as ngVLA requirements.

We pursued two different options: $70 \mathrm{~nm}$ GalliumArsenide (GaAs), metamorphic High-Electron-MobilityTransistor (mHEMT) MMIC LNAs fabricated by the OMMIC foundry, and $35 \mathrm{~nm}$ Indium-Phosphide (InP) HEMT MMIC LNAs fabricated by the Northrop Grumman Corporation (NGC) foundry. Both of these are modifications of the wideband LNAs originally described in [7]. We have tested several versions of these LNAs for broadband performance at the desired 8-48 GHz frequencies in our laboratories at JPL.

\section{MMIC Packaging}

The MMICs were mounted on custom made housing. The input and output of the chip are wirebonded to a 50 microstrip on a 10-mil-thick CuFlon substrate. This microstrip transitions to $2.4 \mathrm{~mm}$ connectors at the housing edges. The bias pads on the chip are wirebonded to single layer bypass capacitors to enhance stability; the DC bias network also includes surface mount capacitors and series resistors mounted on a PCB substrate that also fits inside the LNA housing. An example of the housing assembly is shown in Figure 3.

\section{Noise Measurement Setup}

Noise measurements were performed inside a vacuum dewar that included a radiation shield. Figure 4 shows the setup without the radiation shield. Stainless steel coaxial cables were used at the input and output to maintain thermal isolation. A $20 \mathrm{~dB}$ attenuator was connected at the input of the LNA; this is a common noise measurement technique that uses the Y-factor method but improves the source match of the commercial noise source [7]. Temperature sensors were 


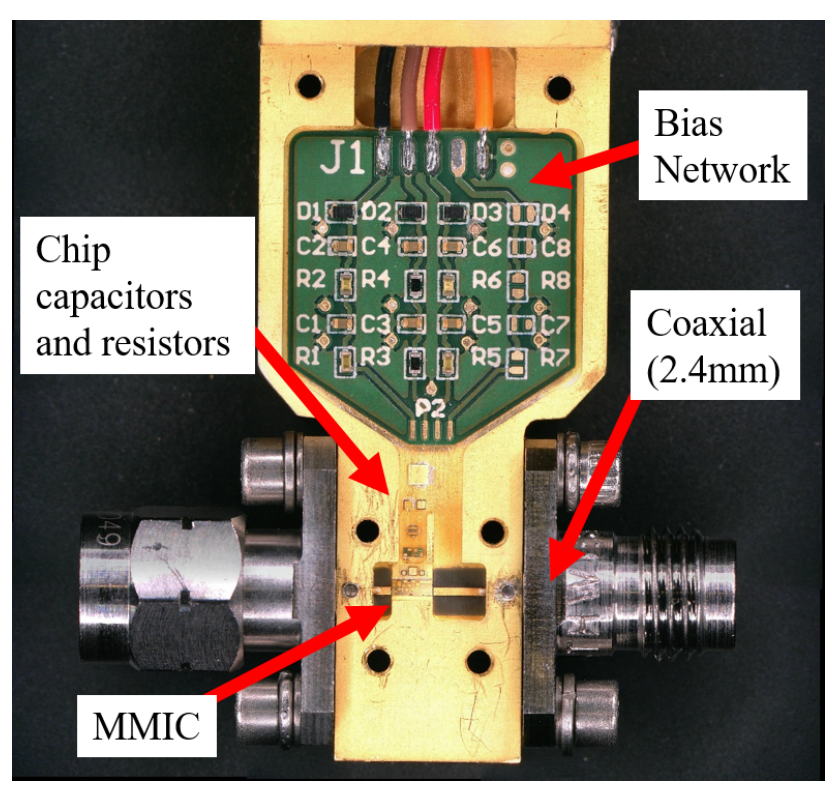

Figure 3. Wideband LNA housing assembly including MMIC, microstrip to coaxial transitions, and bias components.

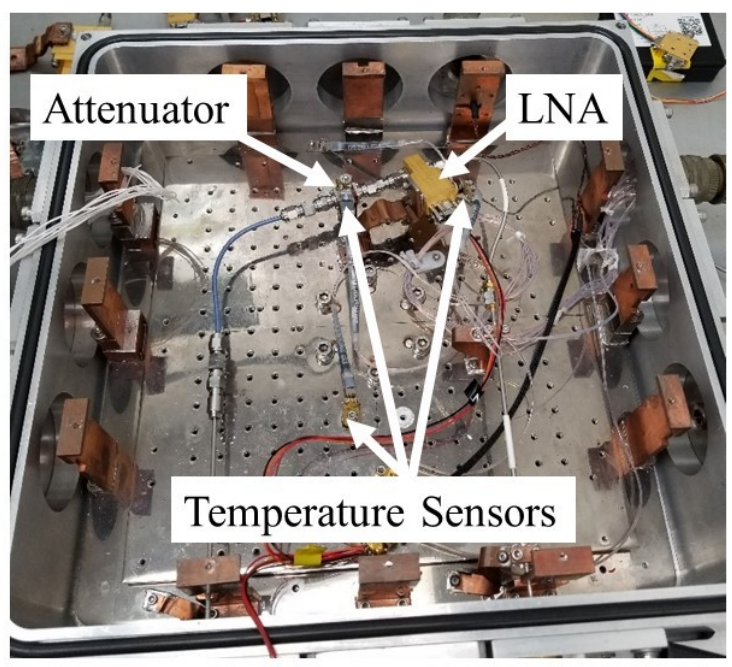

Figure 4. Wideband test bed used for testing the packaged LNAs.

mounted on the LNA, the cold attenuator, and the mounting plate inside the dewar. The LNA housing temperature was $8 \mathrm{~K}$ at the time of the measurements. We used a Keysight 346CK01 noise source and a Keysight UXA-N9040B signal analyzer. All the MMICs were biased for optimum noise.

Usually we performed these measurements with a custom built cold attenuator in which its physical temperature can be mea-sured accurately. In this case we did not have such an attenuator covering this wide bandwidth (measurements without an attenuator caused large ripples in the measurement response due to the mismatch between the $\mathrm{ON}$ and OFF states of the noise source at different frequencies). To circumvent this issue we used an off-theshelf wideband coaxial attenuator and estimated its physical temperature by comparing it with measurements made using a custom made attenuator at lower frequencies $(4-16 \mathrm{GHz})$.

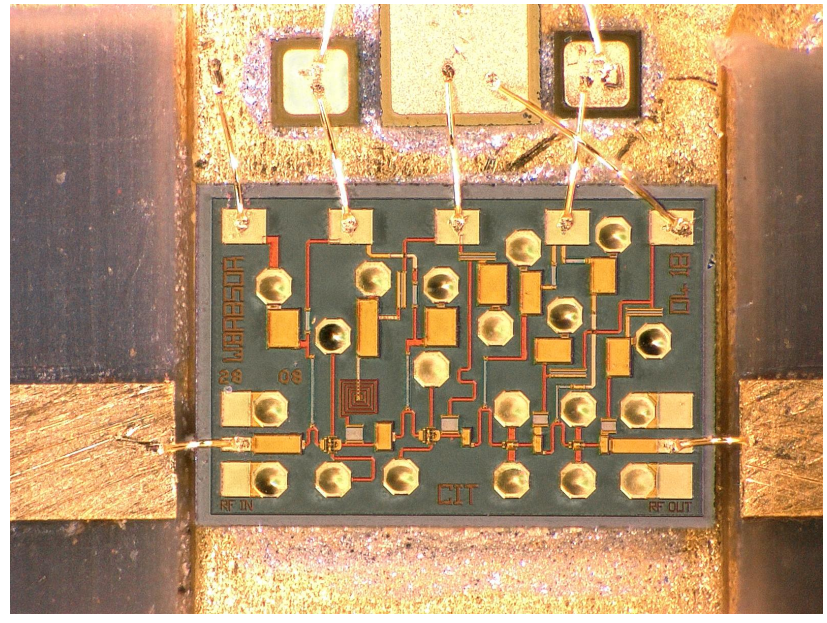

Figure 5. Photomicrograph of a JPL MMIC for 8-48 GHz, manufactured in the OMMIC 70-nm GaAs mHEMT process.

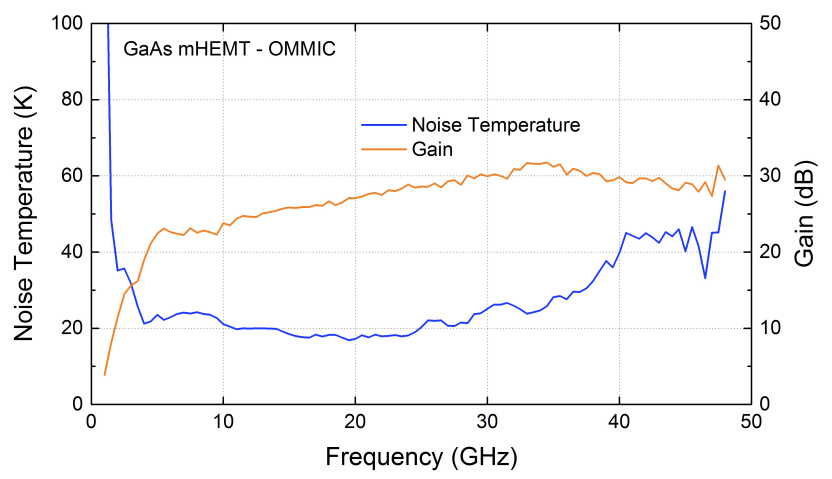

Figure 6. OMMIC LNA and measured data

\section{GaAs mHEMT LNA}

During this project, we evaluated two GaAs m-HEMT lownoise amplifier (LNA) wafers from the foundry OMMIC in France. We tested $70 \mathrm{~nm}$ GaAs m-HEMT low-noise amplifier wafers that we had submitted earlier for fabrication to OMMIC (batch 1). We also received and tested a second wafer run of $70 \mathrm{~nm}$ GaAs m-HEMTs that included 4-finger (WBA850A) and 2-finger (WBA850B) Monolithic Microwave Integrated Circuit (MMIC) LNA chips (batch 2). A close-up picture of one of these LNAs mounted in our custom housing is shown in Figure 5.

We tested three (batch1) 70nm and one (batch2) 4-gate 70nm gate-length mHEMT LNAs. Figure 6 shows results obtained with the 70nm mHEMT LNA from batch2. Note that the LNA noise performance is below $25 \mathrm{~K}$ up to $35 \mathrm{GHz}$ and sharply increases beyond that frequency. The associated gain ranges from 20-30 dB across the band.

\section{InP HEMT LNA}

We also investigated $35 \mathrm{~nm}$ InP HEMT LNAs recently fabricated by Northrop Grumman Company (NGC). These improved MMICs were a result of an earlier optimization design we carried out for 8-50 GHz operation using the NGC process. We built and tested three NGC LNA modules. One of them is shown in Figure 7. In Figure 8 we show typical results obtained with one of these LNAs. Note here that a noise temperature of approximately $20 \mathrm{~K}$ was achieved across 


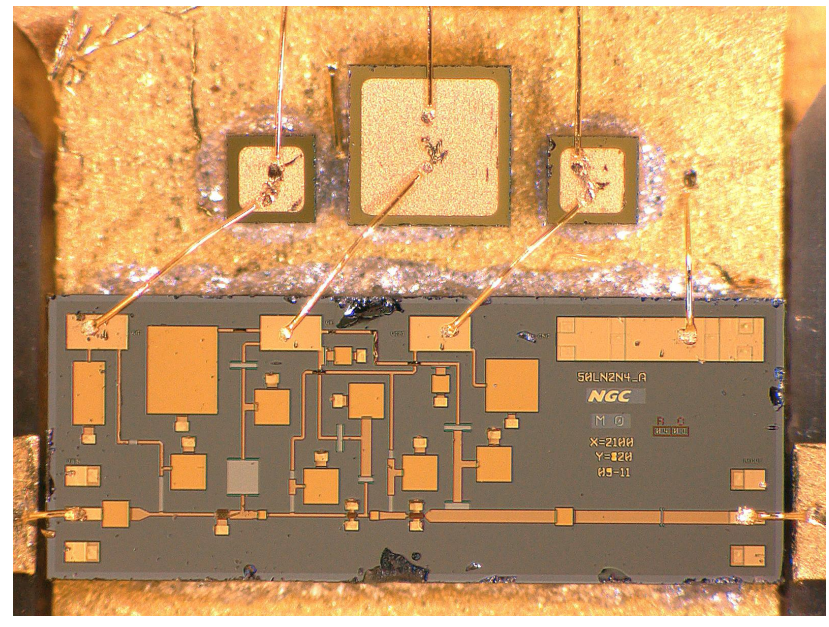

Figure 7. Photomicrograph of a JPL MMIC for 8-48 GHz, manufactured in the NGC $35 \mathrm{~nm}$ InP HEMT process.

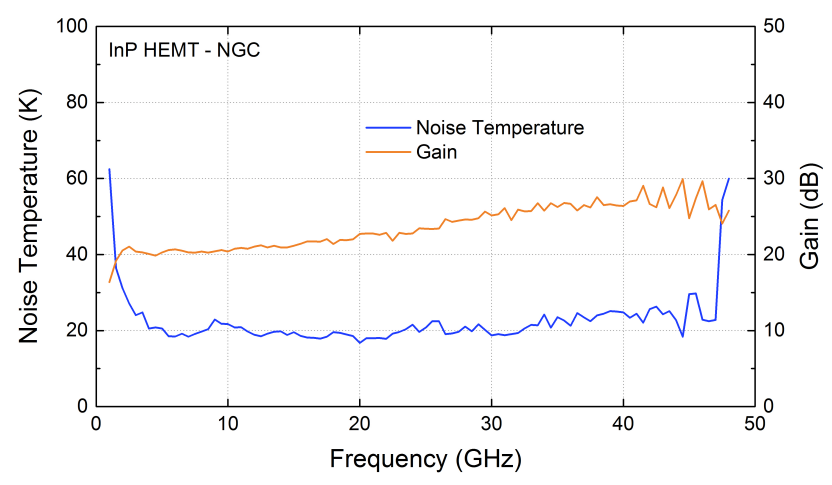

Figure 8. NGC LNA and measured data.

the band. The MMIC gain gradually increases from $20 \mathrm{~dB}$ to about $28 \mathrm{~dB}$ at the high frequency end.

One drawback of our wideband approach is that since all the designs targeted a flat noise response over the bandwidth, the noise at the higher bands is optimized at the expense of the lower bands. Therefore we were not able to achieve state of the art performance at the low edge of the band. Note, however, that the noise performance around $40 \mathrm{GHz}$ is quite remarkable, and very close to our aggressive design goals. In the next section we estimate the total receiver noise temperature based on this LNA results.

\section{NOISE BUdGET}

We have developed a preliminary noise temperature budget that includes the expected contributions of all the ultra-wide band receiver components. These components include the feed, dielectric rod, window, feed to LNA transmission line, LNA and post amplifier. This budget does not include the contribution of the downconverter components. In Table 3 we have tabulated the noise contribution of these components for four frequencies, namely, $8,15,32$ and $48 \mathrm{GHz}$. The receiver temperature, which includes all the components contributions, is referred to as $T_{\mathrm{RX}}$. Note that at the lowest frequency $T_{\mathrm{RX}}=27 \mathrm{~K}$ whereas at the top of the frequency range, $T_{\mathrm{RX}}=38 \mathrm{~K}$. Noteworthy is the fact the main contributor to system noise is the LNA.
Table 3. Receiver System Noise Budget

\begin{tabular}{|c|c|c|c|c|}
\hline Noise (K) & $\mathbf{8 ~ G H z}$ & $\mathbf{1 5} \mathbf{~ G H z}$ & $\mathbf{3 2 ~ G H z}$ & $\mathbf{4 8 ~ G H z}$ \\
\hline \hline Feed & 1 & 2 & 3 & 3 \\
\hline Dielectric Rod & 1 & 1 & 1 & 1 \\
\hline Window & 3 & 3 & 3 & 3 \\
\hline Feed to LNA & 1 & 2 & 3 & 3 \\
\hline LNA & 20 & 20 & 20 & 24 \\
\hline Post Amplifier & 1 & 2 & 3 & 4 \\
\hline \hline $\mathbf{T}_{\mathrm{RX}}(\mathrm{K})$ & $\mathbf{2 7}$ & $\mathbf{3 0}$ & $\mathbf{3 3}$ & $\mathbf{3 8}$ \\
\hline
\end{tabular}

Note that these results, if confirmed by measurements, are comparable to the ones obtained in current JVLA receivers, as shown in Table 1. As with LNAs, the main tradeoff is a relatively poor performance at X-band but remarkable performance at $\mathrm{Ka}$ and $\mathrm{Q}$ band, with a reduced number of components.

\section{FUTURE WORK}

We are currently testing the complete receiver package including a wideband feed and a wideband downconverting stage. Initial test results with the entire receiver package are very encouraging all will be timely published elsewhere. We are also looking into ways to improve the LNA noise performance at X-band without deteriorating excessively the high frequency performance.

\section{Conclusions}

We have presented the development and measurements of wideband low-noise amplifiers based on two different semiconductor processes (GaAs 70nm HEMTS and InP $35 \mathrm{~nm}$ HEMTs) that can be candidates for ngVLA receivers. The LNAs based on InP HEMTs show promising performance, with $20-25 \mathrm{~K}$ noise temperature over the $8-48 \mathrm{GHz}$ bandwidth. We have also shown estimates of the receiver performance that compares well with current JVLA individual band receivers.

\section{ACKNOWLEDGMENTS}

The research was carried out at the Jet Propulsion Laboratory, California Institute of Technology, under a contract with the National Aeronautics and Space Administration. Copyright 2018 California Institute of Technology. U.S. Government sponsorship acknowledged.

\section{REFERENCES}

[1] R. J. Selina et al., "The Next-Generation Very Large Array: a technical overview," Proc. SPIE 10700, Groundbased and Airborne Telescopes VII, 1070010 (6 July 2018).

[2] C. Carilli et al., Next Generation Very Large Array Memo No. 5: Science Working Groups Project Overview, Next Generation Very Large Array Memo Series, October 23, 2015.

[3] D. W. Brown et al., Interagency Telemetry Arraying for Voyager-Neptune Encounter, The Interplanetary Network Progress Report, 42-102, August 15, 1990.

[4] J. Taylor et al., Phoenix Telecommunications, JPL Deep 
Space Communications and Navigation Center of Excellence, August 2010.

[5] S. D. Slobin, 34-m BWG Station Telecommunications Interfaces, DSN Telecommunications Link Design Handbook, DSN No. 810-005, Space Link Interfaces, Module 104, Rev. H, Jet Propulsion Laboratory, Pasadena, California, March 5, 2015.

[6] R. Hayward, The Low-Noise Receiver Systems Developed for the EVLA Project, IEEE Albuquerque Chapter Meeting, October 22, 2014.

[7] A. Akgiray, Ph.D. Dissertation, New Technologies Driving Decade-Bandwidth Radio Astronomy: Quad-Ridged Flared Horn \& Compound-Semiconductor LNAs, Caltech 2013.

[8] A. Dunning, An Ultra-Wideband Dielectrically Loaded Quad-Ridged Feed Horn for Radio Astronomy, Antennas and Propagation in Wireless Communications, 2015 IEEE-APS Topical Conference on IEEE, 2015.

[9] M. Varonen et al., An MMIC Low Noise Design Technique, IEEE Trans. Microw. Theory Techn., vol. 64, no. 3, pp. 826-835, Mar., 2016.

[10] A. Akgiray et al., Noise Measurements of Discrete HEMT Transistors and Application to Wideband Very Low Noise Amplifiers, IEEE Trans. Micro. Theory Techn, vol. 61 , no. 9 pp. $3285-3297$, Sept. 2013

\section{BIOGRAPHY}

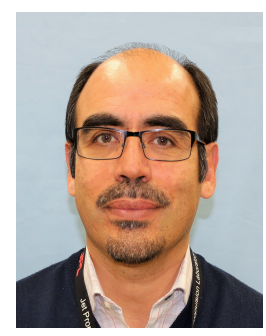

Jose Velazco is the principal investigator for the ultra-wideband receiver for the North America Array program and has over 20 years of experience in carrying out $R \& D$ projects. $\mathrm{Dr} . \mathrm{Ve}-$ lazco has extensive experience in implementing wideband receivers for electronic surveillance applications including wide-open and superheterodyne receivers. Dr. Velazco is the technical supervisor of the Applied Electromagnetics Group at JPL, which develops state-of-the-art high sensitivity cryogenic receivers for NASAs DSN.

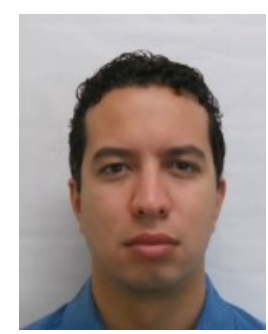

Luis Ledezma is a microwave engineer at the Jet Propulsion Laboratory, California Institute of Technology. He received a MSEE from the University of South Florida in 2011. Prior to joining JPL he was an RF designer at Qorvo for 6 years where he developed MMIC and discrete power amplifiers for the base station market using GaAs HBTs and GaN HEMTs.

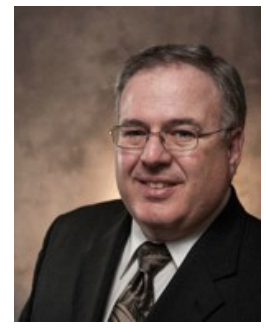

James Bowen has had extensive experience in development of cryogenic LNAs since starting at JPL in 1984. He has worked on development of Low Noise HEMT amplifiers for the Deep Space Network and Radio Science applications. He has also developed and delivered several cryogenic front ends and feed assemblies including control and instrumentation electronics to the DSN and other observatories.

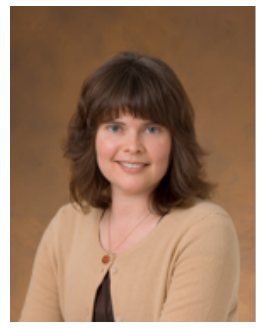

Lorene Samoska received the B.S. degree in Engineering Physics from the University of Illinois in 1989, and the Ph.D. degree in Materials Engineering from the University of California, Santa Barbara, in 1995. She worked as a post-doctoral researcher at UC Santa Barbara in the design and fabrication of state-of-the-art InP HBT microwave digital circuits. She joined JPL in 1998, where she is currently a Principal Engineer involved in the design and testing of 30-600 GHz HEMT MMIC low noise amplifiers and receivers, and power amplifiers for local oscillator sources and transmitters in future space missions.

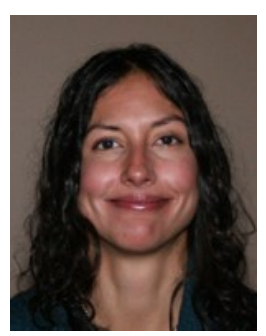

Melissa Soriano is the North America Array Initiative Lead at JPL. Melissa graduated with a B.S. from Caltech, double major in Electrical and Computer Engineering and Business Economics and Management. She also received a Masters in computer science from George Mason University. She has developed real-time software for receivers and antenna array systems used in the Deep Space Network. She was responsible for Direct to Earth communications with Mars Science Laboratory during entry, descent, and landing, and communications with Juno during Jupiter orbit insertion.

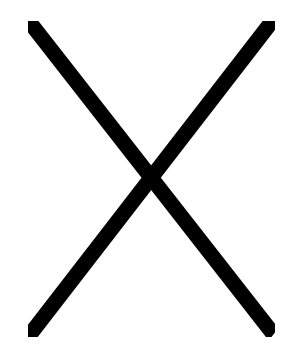

Ahmed Akgiray received the B.S. degree in electrical engineering from Cornell University in 2005, the M.S. degree in electrical engineering from the University of Illinois at Urbana-Champaign in 2007, and the Ph.D. degree in electrical engineering from the Caltech in 2013. Prior to earning his Ph.D. degree, he was with JPL as an RF/Microwave Engineer involved with two spaceflight missions: the Mars Science Laboratory and the Soil Moisture Active Passive (SMAP). He is currently with Ozyegin University, Istanbul, Turkey.

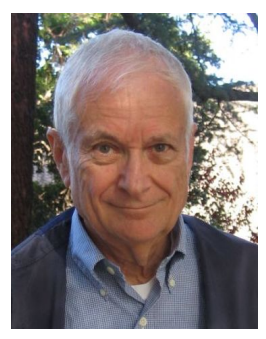

Sander Weinreb received the B.S.E.E. and Ph.D. degrees from MIT in 1958 and 1963, respectively. He is currently a Principal Scientist with the Jet Propulsion Laboratory, California Institute of Technology, Pasadena, CA, USA, and a Sr. Faculty Associate with the California Institute of Technology. Most of his career has been in the administration or development of instrumentation in radio astronomy. His major accomplishments are the introduction of digital correlation techniques into radio astronomy, the discovery of the first cosmic molecular radio spectral line $(\mathrm{OH})$, and the introduction of cryogenic transistor amplifiers to radio astronomy. He has authored or coauthored over 200 publications. His current research activities are in the areas of cryogenic low-noise amplifiers and decade bandwidth antenna feeds. 


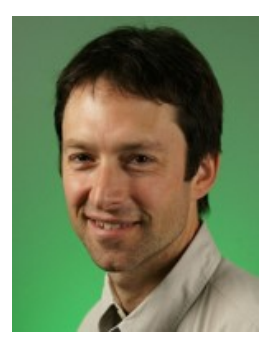

Joseph Lazio is Chief Scientist of the Interplanetary Network Directorate at the Jet Propulsion Laboratory, California Institute of Technology. The Interplanetary Network Directorate manages the Deep Space Network for NASA's Spacecraft Communications and Navigation (SCaN) Division. He received his Ph.D. from Cornell University, was a U.S. National Research Council Research Associate at the U.S. Naval Research Laboratory, and was a Radio Astronomer on the staff of the U.S. NRL, before joining JPL. He was the Deputy Principal Investigator for the proposed Dark Ages Radio Explorer (DARE). He has served as Project Scientist for the Square Kilometre Array (SKA); the Deputy Director of the Lunar University Network for Astrophysics Research (LUNAR); and as Project Scientist for the U.S. Virtual Astronomical Observatory. He also observes routinely with the world's premier ground-based radio telescopes, including the Expanded Very Large Array, the Very Long Baseline Array, the Green Bank Telescope, the Giant Metrewave Radio Telescope, and the Australia Telescope Compact Array. 